\title{
Adaptive computation of gravitational waves from black hole interactions
}

\author{
Philippos Papadopoulos \\ Max-Planck-Institut für Gravitationsphysik, Schlaatzweg 1, 14473 Potsdam, Germany \\ Edward Seidel \\ Max-Planck-Institut für Gravitationsphysik, Schlaatzweg 1, 14473 Potsdam, Germany; \\ National Center for Supercomputing Applications, Beckman Institute, 405 N. Mathews Avenue, Urbana, Illinois 61801; \\ and Departments of Astronomy and Physics, University of Illinois, Urbana, Illinois 61801 \\ Lee Wild \\ Max-Planck-Institut für Gravitationsphysik, Schlaatzweg 1, 14473 Potsdam, Germany \\ (Received 27 February 1998; published 31 August 1998)
}

\begin{abstract}
We construct a class of linear partial differential equations describing general perturbations of non-rotating black holes in 3D Cartesian coordinates. In contrast with the usual approach, a single equation treats all radiative $\ell-m$ modes simultaneously, allowing the study of wave perturbations of black holes with arbitrary 3D structure, as would be present when studying the full set of nonlinear Einstein equations describing a perturbed black hole. This class of equations forms an excellent testbed to explore the computational issues of simulating black spacetimes using a three dimensional adaptive mesh refinement code. Using this code, we present results from the first fully resolved 3D solution of the equations describing perturbed black holes. We discuss both fixed and adaptive mesh refinement, refinement criteria, and the computational savings provided by adaptive techniques in 3D for such model problems of distorted black holes. [S0556-2821(98)01214-4]
\end{abstract}

PACS number(s): 04.25.Dm, 04.25.Nx, 04.30.Db

\section{INTRODUCTION}

The spiraling coalescence of black hole binaries is considered among the most important sources for the upcoming worldwide network of gravitational wave observatories. Essential for the effective detection and interpretation of a coalescing black hole event will be the estimation of the gravitational waveform during the merger [1,2], a very non-linear event, which must be computed by direct numerical integration of the Einstein equations. The amount of reliable detail that can be provided by numerical relativity simulations of such events bears directly on the amount of astrophysical information that can be determined from the observations themselves. The simulations required to study such realistic astrophysical events must be fully three-dimensional. However, the computational requirements for solving the complete set of Einstein equations in 3D are highly non-trivial.

Current 3D black hole simulations produce accurate waveforms for a certain integration time, yet problems with the inner and outer boundaries, and/or poor resolution of certain features, limit the duration of the simulations to a fraction of the physically interesting spacetime. With the exception of very recent simulations based on a 3D characteristic formulation of the equations [3], this is typical of all 3D numerical black hole simulations to date (see, e.g., [4-9]).

From the perspective of gravitational wave astronomy, the availability of adequate resolution in those computations has twofold significance: First, features unique to general relativity would develop in the strong field region near the black holes during the merger, and those effects must be quantitatively captured. This is the problem of correctly modeling the source. Next, the imprint stamped on the radiative part of the metric by the source motion must be given plenty of room and resolution to grow and propagate, up until the point where reading it off will be unambiguous. This is the problem of correctly capturing the gravitational signal. It is obvious from the above discussion, that despite the fact the equations describing black holes can be purely vacuum, there are several scales involved (ultimately deriving from the nature of the imposed initial data).

One can attempt a simple overview of those scales and the corresponding resolution requirements: The shape of the black hole effective potential for gravitational waves, well known from perturbation theory studies [10], can be used as a guiding principle. There are three different regions of importance. Those can be qualitatively appreciated by inspection of Fig. 1. The picture shows the effective black hole potential for generic perturbations (more details are given in Sec. II). The relevant regions span roughly three decades in a logarithmic radial scale, from $0.1 M$ to $100 M$. The inner region has features of the order of $1 M$, i.e., the scale of the horizon for each black hole. Generic strong field regions, modeled in this picture by the potential peak, will encompass the binary and its effective potential, i.e., a domain of roughly $10 \mathrm{M}$. Finally, for a domain extending considerably away from the holes $(\approx 100 M)$, adequate resolution is required for allowing outgoing radiation to form and propagate.

The relative balance of those regions in the resolution budget will depend heavily on the details of the geometrical and numerical formalism. This has led many groups to the proposal and development of concepts which aim (among other things) at reducing the resolution requirements. The inner region $(r<1 M)$, being causally disconnected from the exterior, essentially asks for an effective recipe of no computation, so that the scale that needs to be resolved is 


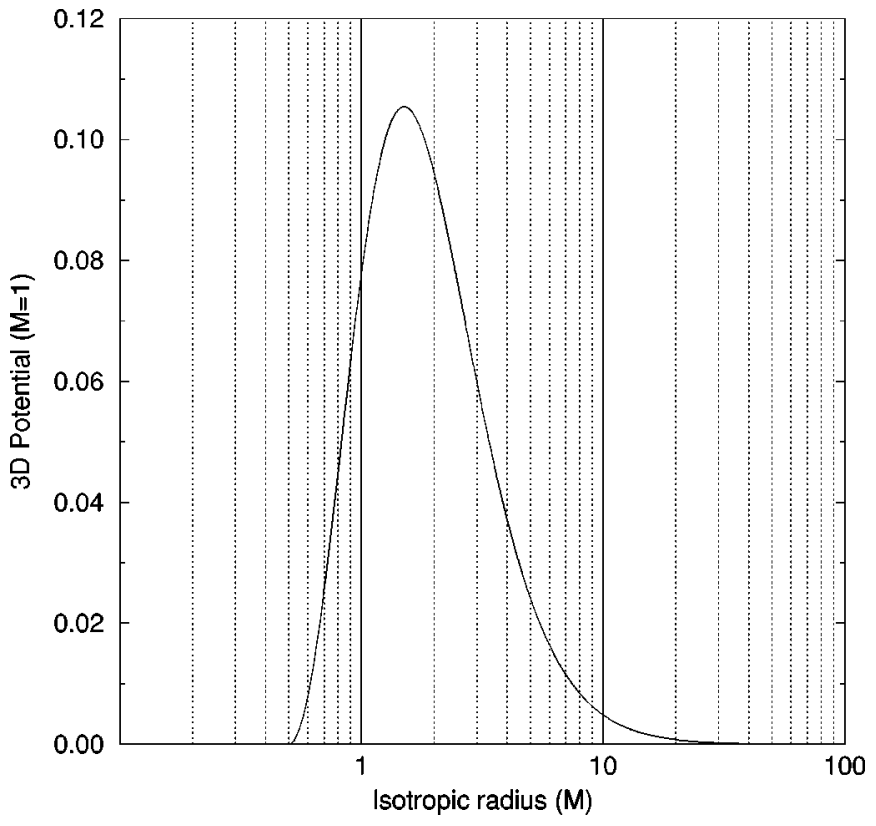

FIG. 1 . The effective potential $a_{3}$ for axial perturbations $\left(K_{1}\right.$ $\left.=-6 M, K_{2}=0\right)$. The interesting range spans three decades in the logarithmic scale. The inner region harbors a one way (ingoing) membrane (the event horizon) which requires adequate resolution in order to prevent reflections from the inner boundary and poor sampling of the rapidly decaying potential. The middle region determines essential features of the outgoing radiation (amplitude, frequency, decay rate). The exterior region sees the wave gradually transform into a radially propagating pulse with a fixed wavelength.

bounded below by the size of the horizons. Apparent horizon boundary conditions (see, e.g. $[11,12]$ ) or characteristic based evolution [3] are the current techniques in this direction. The range of $1 M-10 M$ is thought of as the interesting domain in which the merger drama will unfold, and hence the bulk of the resolution should be provided here. In the exterior region a gradual and subtle transition to the weak field occurs. The domain in the range $10 M-100 M$ may be handled cleanly and efficiently, for example, with a close-in Cauchy-characteristic matching [13]. Other possibilities include the evolution of perturbed spacetimes [14] or other exact approaches as for example, the evolution of conformally compactified equations on hyperboloidal hypersurfaces [15]. Even as those approaches gradually mature, it is important to assess the possibilities of managing the resolution requirements from within the framework of computational science. This paper aims to present some first insights in this direction.

In 3D black hole coalescence simulations, which will likely be performed in 3D Cartesian coordinates, we will need to resolve waves with wavelengths of order $5 M$ or less, where $M$ is the mass of the black hole. Although for Schwarzschild, the fundamental $\ell=2$ normal mode wavelength is $16.8 M$, higher modes, such as $\ell=4$ and above, have wavelengths of $8 M$ and below. More important, for very rapidly rotating Kerr black holes, which are expected to be formed in realistic astrophysical black hole coalescence, the modes are shifted down to significantly shorter wave- lengths $[1,2]$. On the other hand, the size of the black hole is also about $2 M$. As we need of order 20 grid zones to resolve a single wavelength, we very conservatively estimate a required grid resolution of about $\Delta x=\Delta y=\Delta z \approx 0.2 M$. (The best simulations of distorted or colliding black holes in 3D, for which accurate waveforms can be obtained, already use higher resolution, although this is partly needed due to slicing effects $[7,5]$. It is presently unknown whether even higher resolution near the black holes will be required for effective and general apparent horizon boundary conditions.) For simulations of time scales of order $t \propto 10^{2}-10^{3} \mathrm{M}$, which will be required to follow coalescence, the outer boundary will probably be placed at a distance of at least $R \propto 100 M$ from the coalescence, requiring a Cartesian simulation domain of about $200 M$ across. This leads to roughly 1000 grid zones in each dimension, or about $10^{9}$ grid zones in total. As 3D codes to solve the full Einstein equations have typically 100 variables to be stored at each location, and simulations are performed in double precision arithmetic, this leads to a memory requirement of order 1000 Gbytes.

The largest supercomputers available to scientific research communities today have only about $1 / 20$ of this capacity, and machines with such capacity will not be available for some years. Furthermore, if one needs to double the resolution in each direction for a more refined simulation, the memory requirements increase by an order of magnitude. Although such estimates will vary, depending on the ultimate effectiveness of inner or outer boundary treatments, gauge conditions, etc., they indicate that barring some unforeseen simplification, some form of adaptive computation that places resolution only where it is required is not only desirable, but essential for such problems.

The subject of this paper, then, is to elucidate the potential of adaptive methods in three-dimensional black hole simulations. Adaptive mesh refinement (AMR), has been an important development in the last two decades, in applied fields employing large scale numerical computation. It is nevertheless still a frontier area for a wide class of problems. Integration algorithms for hyperbolic 3D partial differential equations, using adaptive finite differences are now increasingly explored [16]. The application of such techniques to general relativistic problems has already shown great promise in one dimensional implementations of the Berger-Oliger AMR method [17]. For example, Choptuik's fundamental work in critical phenomena [18] was enabled by the use of adaptive techniques [19], which were later applied by other groups to similar problems $[20,21,42]$; spherical black hole evolutions were carried out with AMR techniques in [22,25].

More extensive applications of adaptive methods in higher dimensional relativistic problems, are clearly in order. The application of such techniques has primarily been hampered by the complexities associated with computationally implementing the hierarchical tree structure of nested refined subgrids [23] that underlies those methods. In order to circumvent such issues, Wild and Schutz [24] proposed a simpler adaptive mesh refinement data-structure based on hierarchical linked lists. Preliminary tests of this system were applied to model problems involving scalar fields [25].

Building on this work, we adopt here the hierarchical 
linked list (HLL) approach, in its full 3D genre. The still fermenting issue of the correct mathematical approach(es) to the black hole problem, suggests that our exploration of 3D adaptivity in black hole computations would be initially best served by the use of model equations. To this end, we introduce here a class of 3D linear partial differential equations, inspired by the theory of perturbations of black holes, which we then use to study issues pertaining to adaptive computations. There is strong physical motivation for those models, coming from the comparison of perturbative studies of black hole interactions with full non-linear computations, which has shown good agreement [4,26-30] for a large range of currently feasible simulations. The linear model equations we introduce, are completely, even if artificially, threedimensional, i.e., they retain no memory of the angle and time separated equations from which they may arise. Scalar, electromagnetic and axial gravitational perturbations of nonrotating black holes are obtained with the appropriate choice of model parameters. We explore aspects of the models that increase their utility as a calibration tool for the adaptive infrastructure. It is apparent that we exploit here the, by now well developed, interface between perturbation theorists and numerical relativists [31].

We apply fully adaptive mesh refinement techniques for the first time in a 3D relativistic calculation, modeling the dynamics of distorted black holes. We examine a variety of different ways of refining the solution, which we believe are going to be major themes in future applications of AMR to the black hole problem. These include $(a)$ fixed refinement regions that are prescribed in advance, exploiting prior knowledge of the regions that will require high resolution, (b) different geometries of refinement regions, and (c) fully adaptive calculations that follow certain features that develop during the calculation. We discuss the effects of different refinement criteria, and hybrid refinement, which mixes predefined refinement regions with fully adaptive refinement. We show that these techniques can be used very effectively to resolve complex wave structures emitted by systems such as perturbed black holes.

The organization of the paper is as follows: In Sec. II we outline a class of 3D linear partial differential equations that model weak field black hole dynamics. We then study some aspects on the behavior of their solutions, in particular as they introduce the framework in which AMR will be tested. Our adaptive mesh refinement techniques are summarized in Sec. III. In Sec. IV A we present details of AMR simulations that focus on static, prescribed in advance refinement. In Sec. IV B we present dynamical AMR simulations. We close (Sec. V) with a discussion and summary of our results.

Unless stated otherwise, distances refer to the isotropic coordinate system and are expressed in units of the black hole mass $M$.

\section{MODEL EQUATIONS FOR BLACK HOLE PERTURBATIONS}

\section{A. Linear three-dimensional PDE's}

In this section we describe a general class of threedimensional partial differential equations (PDE) that simu- late aspects of black hole perturbations. As our standard example, we start with the Regge-Wheeler equation [32]:

$$
Z_{, r_{*} r_{*}}-Z_{, t t}=\frac{\Delta}{r^{2}}[\ell(\ell+1) r-6 M] Z=V(r) Z,
$$

where $r$ is the Schwarzschild radial coordinate, $\Delta=r(r$ $-2 M), r_{*}$ is a logarithmic "tortoise" coordinate $d r_{*}$ $=r^{2} d r / \Delta$ that relegates the coordinate location of the event horizon to negative infinity, $\ell$ is the spherical harmonic index, and $M$ is the mass of the black hole. This equation describes axial perturbations (odd-parity) of non-rotating black holes, with $Z$ being an appropriate combination of metric perturbations and their derivatives. Note that, because the background is spherically symmetric, the equation is independent of the azimuthal perturbation parameter $m$, hence all perturbations of the same $\ell$ value obey the same equation. As we will see below, it is this particular $\ell$ dependence which allows one to write a single $3 \mathrm{D}$ equation to study the simultaneous excitation of various angular perturbations.

Similar equations, with different potential terms, govern the polar (even-parity) perturbations, as well as the propagation of scalar and electromagnetic waves in the background of a non-rotating hole. Although the equation governing even parity perturbations, first developed by Zerilli, has a more complicated potential, it has identical quasi-normal mode structure, as was shown by Chandrasekhar [10]. However, the $\ell$ dependence of this equation is such that it is difficult or impossible to develop from it a single 3D equation that treats the different angular modes simultaneously. For these reasons, in what follows we will focus on the Regge-Wheeler and similar equations.

In the first step of the construction procedure, we restore the symmetry between spatial directions. This is achieved minimally with the introduction of isotropic coordinates,

$$
\begin{aligned}
& r=\bar{r} A^{2}, \\
& \bar{r}=\sqrt{x^{2}+y^{2}+z^{2}},
\end{aligned}
$$

where $A=1+M / 2 \bar{r}$.

Next, we introduce the field

$$
\phi(t, \bar{r}, \theta, \phi)=\sum_{\ell=0}^{\infty} \sum_{m=-\ell}^{\ell} Z_{\ell m}(t, \bar{r}) Y_{\ell m}(\theta, \phi),
$$

which encodes the aggregate evolution of the separated functions $Z_{\ell m}$. It is then straightforward to establish, that the scaled field variable $\Phi=\phi / \bar{r} A^{2}$ satisfies an equation of the type

$$
\Phi_{, t t}=a_{1}(\overline{\mathbf{x}}) \nabla^{2} \Phi+a_{2}(\overline{\mathbf{x}}) \hat{n} \cdot \bar{\nabla} \Phi+a_{3}(\overline{\mathbf{x}}) \Phi+a_{4} \rho,
$$

where $a_{i}$ are purely radial functions, $\hat{n}$ is the unit radial vector, $\bar{\nabla}$ is the gradient and $\nabla^{2}$ the Laplacian operator, all in Euclidean isotropic three-space. Most notably for our pur- 
poses, in this equation, the $\nabla^{2}$ operator has absorbed the angular operator $L^{2} \phi=-\ell(\ell+1) \phi$ and with it, all dependence on $\ell$.

The coefficient $a_{1}=B^{2} / A^{6}$ determines the coordinate speed of waves $(B=1-M / 2 \bar{r})$. The coefficient $a_{2}=M^{2} B / 2 \bar{r}^{-3} A^{7}$ governs the apparent damping behavior near the horizon. The coefficient $a_{3}$ is the effective potential for the field and determines among others the frequency and decay rate of quasi-normal mode radiation. As mentioned above, perturbations of different spin have different potentials than Eq. (1), the remaining structure of the equation being the same. Following the spirit of [33] we introduce a general effective potential for a non-rotating black hole as

$$
a_{3}=-\frac{B^{2}}{\overline{r^{3} A^{8}}}\left(K_{1}-2 M+\frac{K_{2}}{\bar{r} A^{2}}\right),
$$

where $K_{1}, K_{2}$ are real parameters. The dominant contribution to this potential comes from the Riemann curvature, whose components behave as $M / r^{3}$ for large $r$. Fields of different spin have different quantitative interactions with the curvature potential and this is encoded in the value of $K_{1}$. For fields of spin number $0,1,2$, i.e., scalar, electromagnetic and axial gravitational perturbations, the coefficient $K_{1}$ has values $2 M, 0,-6 M$, respectively. Curvature effects are finite at the horizon which implies that they are redshifted to zero in a static Schwarzschild time slicing [the factor $B^{2}$ in Eq. (5)]. Higher order contributions to the curvature are modeled by the coefficient $K_{2}$, which represents a "deformation" of the potential, e.g., for a charged black hole $K_{2}=4 e^{2}$. A massive scalar field of mass $m$ would have an additional potential term given by $a_{3}=m^{2} B^{2} / A^{2}$, whereas excitations by a source are generically modeled with the term $a_{4} \rho(t, \overline{\mathbf{x}})$, where $a_{4}=B^{2} / A^{2}$. The geometrical factor $a_{4}$ in front of the mass and source terms is redshifting away their contributions near the horizon, and can be obtained by considering the Klein-Gordon equation in the given spacetime and coordinates. Since the background metric is vacuum, the conformally invariant wave equation is also trivially included in the model.

Equation (4) describes the time evolution of general linear fields around non-rotating black holes, in the sense that the evolution of all multipole perturbations can be encoded in this function $\Phi(t, \overline{\mathbf{x}})$ of the four spacetime variables. Initial data for $\Phi$ and $\Phi$ can always be obtained by referring back to Eq. (1), but can also be given arbitrarily, and then correspond to some, probably complicated, superposition of decoupled perturbations. This generality permits the direct study of waves with different wavelengths and arbitrary angular structure. Despite the vector notation in presenting Eq. (4), it should be stressed that the field $\phi$ does not (with the exception of the case $K_{1}=2 M, K_{2}=0$ ), represent a physical scalar in spacetime. Our construction is, in the general case, completely coordinate dependent, and the unit weight factors in Eq. (3) are an arbitrary choice. In the scalar field case, general coordinate transformations of the underlying spacetime (e.g., different time slicings, boosted coordinates) are of course allowed.
To complete the specification of the initial value problem for Eq. (1), we need to supplement Eq. (4) with boundary conditions. With respect to the exterior boundary, we adopt the approach of evolving only the development of the initial Cauchy surface, hence we place our boundary far enough that it will not causally affect the spacetime domain we are considering. For the simulations performed here, the initial data have compact support, which enlarges the domain not influenced by the outer boundary by about one crossing time.

In isotropic coordinates the event horizon is described by the coordinate surface $\bar{r}_{H}(x, y, z)=M / 2$. The use of Schwarzschild slicing implies that the lapse function has a zero there. The effects of angular momentum and curvature are rapidly damped (see for example [10]) and the effective black hole potential decays exponentially in $r_{*}$. Imposing an ingoing wave type condition

$$
Z_{, t}-Z_{, r *}=0
$$

at some inner timelike world-tube at radius $r_{*}^{B}$ is applicable for any equation of the form of Eq. (1). The transformation of condition (6) to the coordinates and variables of our model equation yields

$$
\Phi_{, t}=\frac{B}{A^{3} \bar{r}}\left(\overline{\mathbf{x}} \cdot \bar{\nabla} \Phi+\frac{B}{A} \Phi\right) .
$$

Condition (7) becomes equivalent to a static horizon condition $(\Phi=0)$, when applied in the limit $\bar{r}_{B} \rightarrow \bar{r}_{H}$, but is actually extremely accurate, when applied at a timelike worldtube that is inside the decay width of the potential, as it falls from its peak value at $\bar{r}_{P} \approx 2$. This fact has been both extensively tested and used in the literature.

\section{B. The nature of the solutions}

Here we touch upon issues regarding the model equation (4), especially as they apply to our adaptive computations.

The merger waveform, due to the final interactions of the black holes as they coalesce, will be an important component of the detected signal, which when detected can presumably reveal intricate details of the coalescence process. Even the broad features of the merger waveforms are uncertain at present (and may require techniques such as those we are developing and testing here to compute).

As we have emphasized, the later stages of binary black hole mergers lead to highly distorted black holes that can be treated surprisingly well by perturbation theory. Even the entire collision process has proved amenable to a perturbative treatment in certain regimes. Through this large body of work, the following picture has emerged: during the very late stages of a binary black hole merger, the highly distorted black hole formed in the process rings down to its Kerr form through a progression of regular, damped oscillations, with the least damped quasinormal mode quickly dominating the picture. The frequency (wavelength) of the slowest damped mode is an important feature of the solution in the ring-down phase. Fully nonlinear simulations of such distorted rotating black holes have been performed, and the $(m=0)$ quasi- 


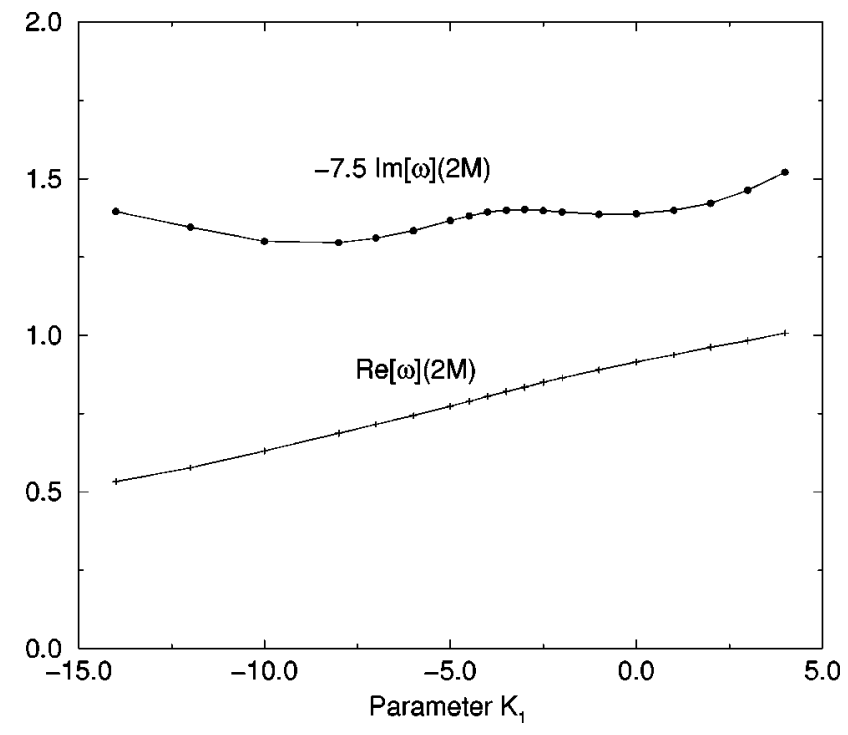

FIG. 2. Graphical representation of the frequency and decay rate of the slowest damped mode for potentials of the type $a_{3}$. Here $l$ $=2$ and the parameter $K_{2}$ has been set to zero. The derived values for $K_{1}=-6 M, 0,2 M$ agree well with values tabulated in the literature.

normal modes are found to be the dominant feature of the waveforms [34].

For full 3D black hole coalescence, leading to a rapidly rotating black hole, at late times the $\ell=m=2$ mode is expected to dominate, having a much higher frequency than its Schwarzschild counterpart (perhaps two to three times higher). Hence, for realistic 3D simulations, the resolution requirements will be higher than what would be indicated by a naive treatment of Schwarzschild perturbations. A powerful approach to modeling the ringing phase of a rotating black hole would be the use of equations based on curvature perturbations. Recently, certain members of the Teukolsky family of equations have been integrated numerically as a $2+1$ problem $[35,36]$. As some aspects of the numerical integration of those equations e.g., coordinate systems, higher dimensional simulations, are now under investigation, we chose here to base our model in the more thoroughly understood dynamics of potential type equations.

The potential of Eq. (4) can be modified to allow the study of a larger range of ringing wavelengths. To this end, we have performed a numerical study of the slowest damped modes of Eq. (4) for various angular multipoles, using 1D versions of the equations, as such a catalog is convenient for calibrating the three-dimensional code. The behavior of the real and imaginary parts of the quasi-normal mode (QNM) frequency are rather interesting. We mention for example that the slowest damped mode of the equation has a real frequency that is a monotonic function of $K_{1}$, for any fixed $K_{2}$. The imaginary part (decay rate) has an oscillatory dependence on $K_{1}$. Different values of $K_{2}$ tend to introduce an overall shift on the dependence of $\omega$ on $K_{1}$. Figure 2 provides a convenient calibration of the slowest damped mode wavelength and decay rate.

\section{1-Dimensional considerations}

In this work we use the structure of the QNM's as an important diagnostic tool for the efficiency, flexibility and accuracy of the AMR suite. For convenient calibration we use evolutions computed with one-dimensional codes, both in the well behaved tortoise coordinate, which approaches $-\infty$ at the horizon, and the isotropic radial coordinate, which goes to a finite value of $r=0.5$. The tortoise coordinate is most naturally adapted to the problem, accounting exactly for the infinite blue shift of a wave as it approaches the horizon. If an ingoing wave is resolved on an equally spaced grid in the tortoise coordinate near the peak of the potential, it will remain so as it propagates towards the horizon, even as its wave is highly blue shifted physically. The tortoise coordinate can be considered as a natural adaptive mesh for the problem, and the 1D code in this formulation can easily give accurate results for a given $\ell$-mode.

On the other hand, the isotropic coordinate description provides important clues for the minimal amount of resolution required for the $3 \mathrm{D}$ code, as it is directly related to the 3D Cartesian coordinates. In this coordinate, an ingoing wave will be shifted to much shorter wavelengths as it approaches the horizon, and hence the wave will very rapidly become difficult to resolve as it propagates in. This is an effect of the Schwarzschild slicing we are using in the model equation. This effect can be seen alternatively by considering the reduction of the wave speed as one approaches the horizon: with the inner boundary at an isotropic radius of $r$ $=0.6$ (Schwarzschild radius 2.016 ), the speed drops by thirtyfold to about 0.027 .

In spite of these considerations, one might think that the treatment of the equation near the horizon would not necessarily have important effects on the waves propagating far from the black hole, for several reasons. First, waves should be essentially ingoing in this area, second, any reflections due to poor treatment here will be rapidly redshifted as they climb out of the black hole potential, and finally, the potential barrier in Fig. 1 acts to protect the outside observer. However, it turns out that significant fractions of the emitted signal are influenced strongly by the inner region near the horizon, i.e., $\bar{r}_{B}<\bar{r}<\bar{r}_{P}$. In particular, poor resolution of the inner region and inner boundary can have very adverse effects on the final waveform as shown below.

We demonstrate these points with a series of 1D simulations for an $\ell=2$ wave in Fig. 3. We compare three simulations that illustrate the importance of proper treatment near the horizon. Initial data consist of a Gaussian packet localized to a region outside the potential barrier with vanishing initial time derivative. The parameters in the potential correspond to the standard Regge-Wheeler potential. The integration domain extends from an isotropic radius of $r=0.6\left(r_{*}\right.$ $=-7.56)$, to $r=100$. The tortoise based evolution attains adequate accuracy for our purposes already for resolution $N=200$, where $N$ is the number of grid points $\left(\Delta r_{*}=0.5\right)$; for comparison purposes we use a more than sufficient $N$ $=2000$ grid. The solid line in both panels of Fig. 3 shows the signal recorded at a radius $r=10 M$, as obtained with the tortoise code. After an initial burst of waves, the quasinormal 

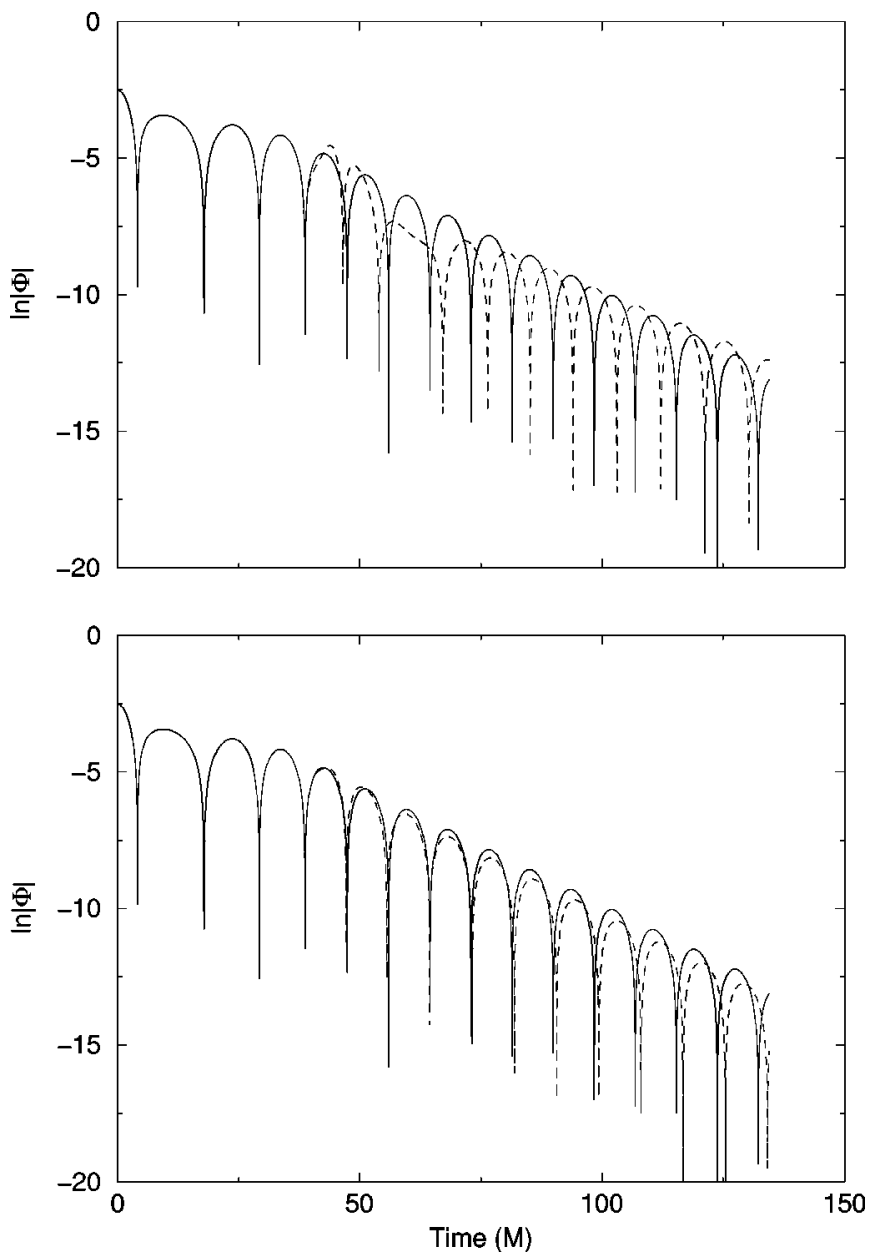

FIG. 3. We show 1D results for a Gaussian wave packet hitting the black hole Regge-Wheeler potential. The solid line in both panels shows the waveform measured at $r=10 M$, obtained with an equally spaced grid (in tortoise coordinate) of $\Delta r_{*}=0.05$, or $N$ $=2000$. The dashed lines show results obtained with the isotropic code using a fixed resolution of $\Delta r=0.05$ (upper panel) and $\Delta r$ $=0.02$ (lower panel), which corresponds to $N=2000$ and $N$ $=5000$, respectively. The ingoing boundary condition is always applied at the same radius, $r=0.6$. For large $r$, the tortoise and isotropic coordinates are very similar, and therefore meager resolutions of the order of 0.5 would be adequate to resolve the burst phase (the first part of the signal, which overlaps perfectly). But near the horizon, the wave is woefully under-resolved in isotropic coordinates, even with a grid that is ten times finer $(\Delta r=0.05)$, which shows its wrath on the waveform at late times, i.e., when the under-resolved region near the horizon makes causal contact with the observer. With increased resolution (lower panel) there is marked improvement, and eventual convergence of the QNM signal.

modes of the black hole dominate, and are well resolved. We use this as our fiducial run against which we compare other simulations.

We now examine results obtained with the isotropic radius, which gives an indication of the problems that will be encountered in 3D. In Fig. 3 the dashed lines show results obtained with the isotropic code using a fixed resolution of $\Delta r=0.05$ (upper panel) and $\Delta r=0.02$ (lower panel), which corresponds to $N=2000$ and $N=5000$, respectively. The ingoing boundary condition is always applied at the same radius, $r=0.6$. For large $r$, the tortoise and isotropic coordinates are very similar, and therefore meager resolutions of the order of 0.5 would be adequate to resolve the burst phase (the first part of the signal, which overlaps perfectly). But near the horizon, the wave is woefully under-resolved in isotropic coordinates, even with a grid that is ten times finer $(\Delta r=0.05)$, which shows its wrath on the waveform at late times, i.e., when the under-resolved region near the horizon makes causal contact with the observer. With increased resolution (lower panel), there is marked improvement, and near convergence to the true QNM signal. In contrast the solution for $\Delta r=0.05$ (upper panel) illustrates the subtle effect on the phase and frequency of the QNM, even for what would be thought of as a very fine resolution. The frequency shift in the QNM suggests that the poor resolution of the horizon region modifies the effective potential experienced by the gravitational wave. This argument is made more plausible by the fact that, even in flat space, a reflecting potential near the origin supports a finite number of QN-modes, see $[37,38]$.

The severity of the effect suggests we have a first rate test problem on which to apply AMR techniques. In order to obtain a sufficiently resolved simulation with a uniform grid we had to use more than 25 times the basic tortoise resolution of $N=200$. In this case, it is possible in $1 \mathrm{D}$, but in $3 \mathrm{D}$ such a factor would lead to $25^{3} N^{3}$ ! We will turn into the handling of this behavior in the next section.

Those adverse effects of poor resolution around the horizon suggest that this is an issue to be kept in mind in more general simulations. In non-linear numerical relativity simulations, the time slicing is dynamic and generally has a finite lapse at the horizon. Despite the reduced blueshift experienced by a wave (the most demanding factor), other physical factors also compete for resolution: (a) the geometrical volume factor (since we are using Cartesian coordinates); (b) the need for sufficient sampling of the inner part of the potential (see Fig. 1) in order to capture accurately the correct scattering and development of the quasinormal modes; and (c) the need to guarantee convergence for any type of analytic condition near an excised horizon area. Hence, also in implementations of apparent horizon boundary conditions (AHBC), one will need to be careful to ensure that the waves going in at the horizon be properly resolved, and that the boundary condition allows ingoing waves to propagate off the grid, if one is to get the black hole dynamics correct. To date, even in successful implementations of AHBC, only the longitudinal part of the spacetime has been tested. These AHBC simulations need to be applied to spacetimes with dynamic black holes for which waveforms can be extracted, such as those recently studied in Refs. $[6,7,4,5]$ to ensure that the low amplitude waves are not adversely affected by the treatment of the spacetime near the black hole.

\section{THE NUMERICAL ALGORITHM: MULTI-DIMENSIONAL HIERARCHICAL LINKED LIST AMR}

Conventional adaptive mesh refinement assumes a data structure based on a hierarchical tree of embedded refined 
sub-grids ( [17]). In one dimension this hierarchy is simple and efficient-each sub-grid is just a spatial interval demarcated by an upper and lower bound-however in multidimensions, one must first demarcate regions of refinement into clusters and then for each cluster a refined rectilinear sub-grid must be placed over it (for example [39]). The quality by which one is able to match the desired refinement topology with rectilinear boxes is given by an input parameter-the clustering efficiency-which when set to $100 \%$ produces a minimal refinement topology. However, depending on the refinement topology itself, this may mean the production, storage and management of many thousands of "little boxes." This number of boxes (and hence their associated computational cost of management) can be reduced considerably by reducing the clustering efficiency, but this inevitably over refines the computational domain and thus increasing once more the computational cost this time of integrating the solution. Therefore, usually a balance needs to be attained between the increased computational overheads required to achieve and maintain a grid hierarchy with a $100 \%$ clustering efficiency, hence many boxes, but no over-refinement of the computational domain as compared to a reduced clustering efficiency, hence less refined boxes, but over-refinement of the domain.

To avoid such scenarios we have instead adopted the AMR method proposed by Wild and Schutz [24]. Here they build mesh refinement that precisely matches the desired topological requirements, but without clustering algorithms.

Their method centers on the representation of mesh refinement by a simple hierarchy of refinement levels

$$
\mathcal{R}_{0} \cup \mathcal{R}_{1} \cup \mathcal{R}_{2} \cup \ldots \mathcal{R}_{l} . . \cup \mathcal{R}_{\text {TotalLevels }},
$$

where $\mathcal{R}$ is a uniform distribution of grid points of arbitrary topology and the subscript indicates the magnitude of the spatial and temporal resolution such that $\mathcal{R}_{0}$ has the least resolution and $\mathcal{R}_{\text {TotalLevels }}$ the greatest. For neighboring refinement levels $\mathcal{R}_{l}$ and $\mathcal{R}_{l+1}$, the boundaries of the former always contain those of the latter (i.e., $\mathcal{R}_{l+1} \in \mathcal{R}_{l}$ ) and their spatial and temporal resolutions differ by an integer refinement factor $\eta$ we choose to be two.

To construct each refinement level, in 3D Cartesian coordinates, three sets of one dimensional double linked lists are required (one set along each of $x, y$ and $z$-dimensions.) These lists are in turn built from a data structure (called nodes) which contain:

A hexahedron of grid points.

Six sibling pointers (two for each dimension) for the double links between neighboring nodes.

Eight offspring pointers to allow each grid point present to spawn a new, offspring, node.

One parental pointer to identify the node it is refining.

A flag to indicate whether it requires refinement or not.

An example of this type of mesh topology construct is shown in Fig. 4. Here the coarse grid $\mathcal{R}_{0}$ (large cubes) consists of a $4 \times 3 \times 2$ lattice of nodes and therefore represents a $8 \times 6 \times 4$ lattice of grid points (assuming a refinement factor $\eta=2$.) The smaller nodes are refinement level $\mathcal{R}_{1}$. Here it is clear to see why eight are required to refine a parental node.

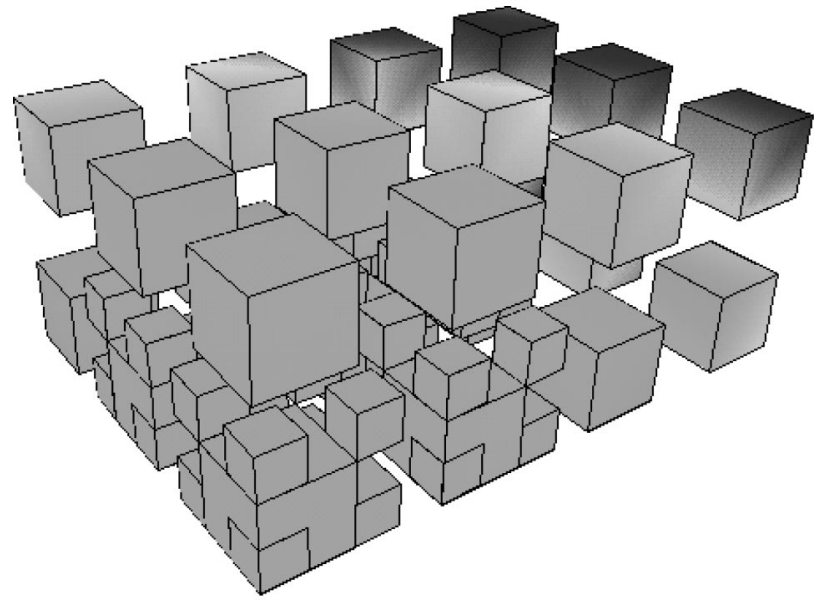

FIG. 4. 3D mesh refinement using nodes. The large cubes (nodes) represent a $8 \times 6 \times 4$ lattice of grid points that make up the base grid $\mathcal{R}_{0}$. The smaller nodes are the refinement level $\mathcal{R}_{1}$.

To evolve the refinement hierarchy, we use a modified Berger-Oliger algorithm [24]. Here the concept of each refinement level being integrated by one time step and then only being integrated again once all higher refinement levels have been integrated to the same temporal point, remains unaltered. However, whenever neighboring refinement levels $\mathcal{R}_{l}$ and $\mathcal{R}_{l+1}$ are temporally coincident, then only the subset $\mathcal{R}_{l} \cap \mathcal{R}_{l+1}$ of the $\mathcal{R}_{l}$ solution that can numerically influence the boundary of $\mathcal{R}_{l+1}$ is replaced by that in $\mathcal{R}_{l+1}$, as compared to the Berger-Oliger approach which injects the complete $\mathcal{R}_{l+1}$ solution onto $\mathcal{R}_{l}$. This much reduced interrefinement communication can help performance on parallel computing platforms in situations, where the domain decomposition strategy for distributing the mesh refinement's topology across processors does not ensure regions of refinement are aligned with their parental region. Thus, potentially expensive inter-processor communications are reduced from that of a refinement level's volume to just its surface area.

To integrate the solution on each refinement level, the AMR method (written in C) traverses all linked lists in the $x$-dimension-one node at a time-by using the appropriate pointers. For each node the necessary finite difference stencil is read from memory and passed to a separate program (written in Fortran90). Here, the solution for the new time level is computed and then returned to the AMR code to be stored (in this way AMR is independent from the simulation-it choreographs the topology of the mesh and the storage of the solution therein, nothing more.)

The mesh is modified periodically to ensure its topology satisfies that of the evolving solution. This process is always carried out from $\mathcal{R}_{\text {TotalLevels }}$ downwards, such that for each refinement level nodes are checked against the refinement criteria to determine whether offspring nodes need to be created or destroyed.

The AMR method is made parallel on shared memory architectures using a simple one dimensional domain decomposition strategy whereby the total number of lists in the direction of traversal are distributed evenly between the available processors [25,43]. Although simple, such a 
TABLE I. The first column refers to the refinement level, which is bounded in the outside by the radial value of the second column. The resolution of each level (refinement by factors of two) is given in the third column. The innermost grid point at which a given level approximates the inner boundary is shown in the fourth column.

Refinement level Outer bound (M) Resolution (M) Inner bound

\begin{tabular}{cccc}
\hline$r_{1}$ & 10.0 & 0.25 & 0.75 \\
$r_{2}$ & 5.0 & 0.125 & 0.625 \\
$r_{3}$ & 2.5 & 0.0625 & 0.625 \\
$r_{4}$ & 1.5 & 0.03125 & 0.625 \\
$r_{5}$ & 1.0 & 0.015625 & 0.609375 \\
$r_{6}$ & 0.75 & 0.0078125 & 0.6015625 \\
$r_{7}$ & 0.62 & 0.0039860 & 0.6015625 \\
\hline
\end{tabular}

scheme should not be under-estimated, since it has the advantage of ensuring every processor has a $100 \%$ work load during the Berger-Oliger integration cycle (a great advantage when the workload per grid point is high as is the case, when considering the fully relativistic Einstein equations.) Additionally, it introduces a minimal change to the code: one must now ensure each processor maintains temporal synchronization during the Berger-Oliger integration cycle [17].

The discretization of the model equation within the AMR framework is straightforward and is done using a three-level leapfrog scheme. Interior points are updated with the regular nine-point stencil, which is for the centered differencing of first and second order derivatives. Boundary points are masked and discretized with sidewise differences.

\section{3D ADAPTED COMPUTATIONS}

\section{A. Prescribed mesh refinement}

It is clear that in a 3D integration of the model equation some sort of adaptive grid is required at least in the region near the horizon. We have as our guide the model 1D problem above, where we saw in Sec. II C how critical it was to resolve the region around the horizon. Equal spacing in the tortoise coordinate gives constant resolution coverage of a blueshifting wave as it approaches the horizon, which translates into increasing resolution requirements in the isotropic coordinate, and hence in the Cartesian coordinates. Indeed unigrid (i.e., single uniform spacing) 3D Cartesian evolutions with up to $300^{3}$ grids covering a region from the horizon to a distance $r \approx 100 M$ produce woefully inadequate waveforms, as expected from the 1D considerations above. The question we address next is how to provide the adaption.

We first investigate whether one can use certain prior knowledge of a system to construct an adapted predefined mesh appropriate to the problem. We hence prescribe successive refinement layers around the horizon, whose topology and location remains constant throughout the evolution. This approach is a powerful and efficient use of the AMR infrastructure in three dimensions, which is easily combined with dynamic refinement in other parts of the domain.

Here we illustrate this technique with an example of seven successive layers (Table I) of refinement around the

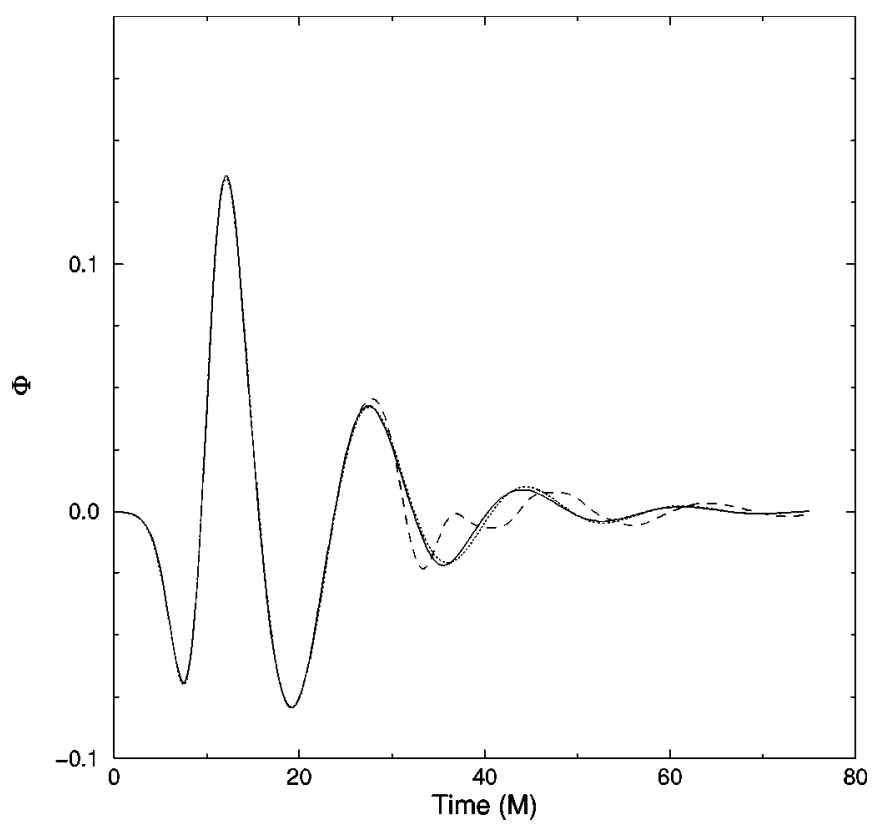

FIG. 5. We show waveforms obtained for different levels of fixed refinement in the interior region near the horizon, and its effects on the quasi-normal mode structure. The solid line is the 1D result, which is progressively attained using three refinement layers (dashed line) and seven refinement layers (dotted line). Poor resolution leads to under-sampling of the inner part of the potential which combined with reflections from the ingoing boundary condition generates an effective numerical potential with different frequency and decay rate. Figure 6 illustrates this point more clearly with the use of logarithmic scale for $\Phi$.

black hole horizon, which were motivated by studies of the $1 \mathrm{D}$ equation in isotropic coordinates. A valuable guide is the study of two radially ingoing null geodesics $\bar{r}_{1}(t), \bar{r}_{2}(t)$, and correspondingly the allocation of resolution so as to preserve roughly the number of grid points in-between the geodesics as a function of radius. To test this nested grid structure, we set up an initially time symmetric quadrupole Gaussian pulse in the Regge-Wheeler function $\Phi$ located at $10 M$. Specifically, the initial data takes the form:

$$
\begin{aligned}
& \Phi=e^{-\kappa\left(\bar{r}-\bar{r}_{c}\right)^{2}} R(\theta, \phi), \\
& \dot{\Phi}=0,
\end{aligned}
$$

where $\kappa$ and $\bar{r}_{c}$ control the width and location of the shell, and $R(\theta, \phi)$ sets an appropriate angular dependence, which for our standard comparison with the 1D Regge-Wheeler equation, we adopt to be a quadrupole:

$$
R(\theta, \phi)=\sin ^{2} \theta \cos (2 \phi+\pi / 2)
$$

(the rotated form of $R$ in this particular case is explained in the Appendix).

The accuracy in resolving the QNM structure using such nested refinement levels is demonstrated in Figs. 5,6. Figure 5 shows results in linear scales, showing the familiar ringing waveform, while Fig. 6 shows the same simulation in a loga- 


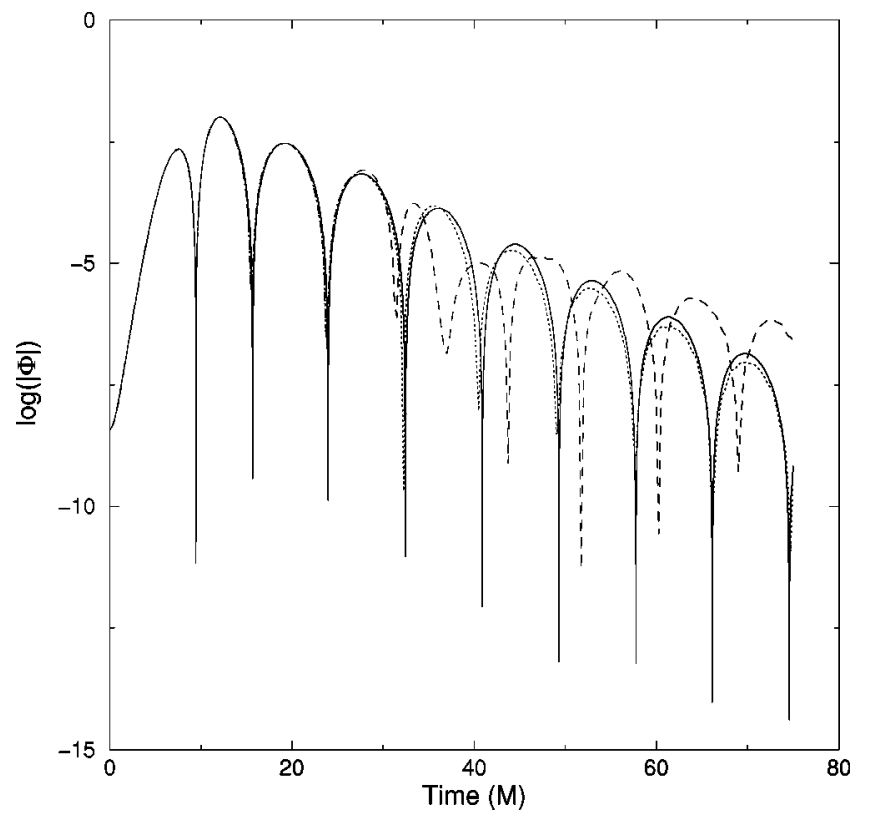

FIG. 6. Logarithmic plot illustrating accuracy issues related to the generation of QNM signals. The time signal is extracted around $3 \mathrm{M}$ and is compared with the corresponding one-dimensional result (solid line). The base grid has a resolution of $0.5 \mathrm{M}$, which is too coarse to resolve the black hole potential and hence produces a large reflected wave (not shown). Using three refinement layers (finest level at $0.0625 \mathrm{M}$, dashed line) considerably reduces the reflections, but cannot reproduce most of the QNM structure. Further refinement (dotted line) to seven levels $(0.0039062 \mathrm{M})$ captures the correct QNM frequency and decay rate over the whole domain of interest.

rithmic scale, to bring out different aspects of the results. The comparison is done at the peak of the potential (similar signals are obtained e.g., at $r=10 M)$. The base grid is at $\Delta x=\Delta y=\Delta z=0.5 M$ resolution and extends to $r=50 M$.

The solid lines are 1D results, using about $N=1000$ radial points using the tortoise code. The 3D solution converges to the correct (1D) one as more layers of refinement are added (the coarse grid evolution is too erroneous to display). Using three refinement layers (finest level at $\Delta x=0.0625 M$, dashed line) considerably reduces the reflections, but still has a serious effect on most of the QNM structure. Further refinement (dotted line) to seven levels $(0.0039062 \mathrm{M})$ captures the correct QNM frequency and decay rate, over orders of magnitude in the decay. Further enlargement of the thickness of the layers reduces the amplitude loss at the expense, of course, of computational time.

These results have been obtained with refinement levels using nested spheres, which naturally adapt to the geometry of a central black hole. Even though the underlying coordinate system being used is Cartesian, by using linked lists this structure is still straightforward and natural to create. With more traditional grid structures, if one attempted nested refinement levels it might be easier to create nested cubes. With the linked lists we can also easily create such refinement structures and study their effect on the results. Using this nested box construction, we have reproduced results for various refinement layers. The results are excellent in both cases, but there is a slight improvement in the quality of the modes, when using boxes with width equal to the diameter of the corresponding sphere. This is expected, given the considerably larger refinement volume introduced by nested cubes. This result suggests that using linked lists to construct spheres within spheres, one can match the accuracy of the nested cube waveforms, but using $\pi / 6$ of the memory. This non-negligible saving is inherent to the linked list approach, not to AMR, and could equally well be applied to the construction of uniform grid codes. Furthermore, in addition to handling irregular outer boundaries, linked lists are highly amenable to the construction of an internal boundary, e.g., within a black hole's horizon as shown here.

Longer evolutions of the same initial data reveal an interesting phenomenon associated with the presence of different refinement levels. Observers inside a given refinement level will observe numerical reflections arising at the refinement level boundary with the next coarsest grid. In our simulations those reflections were well below the interesting signal levels, but this may change in non-linear simulations with more meager resolutions, or refinement factors larger than two.

To summarize the results of this section, we note that the essential dynamics of a black hole in the linear regime was resolved with a fixed, refined mesh structure. In this approach we use knowledge of the system to place appropriate refinement levels where they will be needed. We also found that the underlying geometry of the refinement levels can be flexible, i.e., we found that nested boxes and nested spheres both do an excellent job of resolving the calculation, although the nested spheres allow considerable memory savings. We note, however, that fixed refinement must be used with care: if highly resolved waves are allowed to propagate into regions that are unable to resolve a wavelength, spurious reflections. (See Wild [25] or Wild and Schutz [24] for a detailed analysis of reflection and transmission properties of waves crossing various grid interfaces, along with strategies to handle such problems). Extending the refined domain as the generated signal propagates outwards, away from the highly resolved horizon region, is a natural application for dynamic mesh refinement, to which we turn next.

\section{B. Dynamic mesh refinement}

Using our model equation we also explored the issue of outgoing waves - with wavelengths typical of black hole QNM's-propagating outwards on an initially coarse grid, which would not provide adequate resolution if not refined. Depending on the initial burst of waves hitting the black hole, different outgoing radiation patterns and wavelengths may emerge, which cannot be predicted ahead of time. We now examine approaches for supplying the necessary resolution to these waves, which must be tracked and resolved as they propagate. In what follows we maintain the static refinement necessary to resolve the region around the horizon (including all seven levels listed in Table I), and explore different methods to track the waves as they propagate to large distances from the black hole. In this "hybrid" refinement strategy, we allow the adapted grids to follow the waves wherever they go, both away from the hole and into the highly refined region near the hole. 
A crucial aspect of AMR is the choice of refinement criteria. A common general purpose estimator for dynamic refinement is the intrinsic truncation error estimate. Such an estimate of the truncation error of a numerical solution, $\tau$, can be defined as

$$
\tau \propto\left|\mathcal{Q}_{\Delta s, \Delta t}^{2} \phi-\mathcal{Q}_{2 \Delta s, 2 \Delta t} \phi\right|,
$$

where $\mathcal{Q}$ is the finite difference evolution operator used to integrate the solution and $\Delta s, \Delta t$ are the spatial and temporal resolutions, respectively. Thus, the truncation error is computed by taking the difference of the solution obtained using two regular time steps of $\Delta t$ for a spatial resolution $\Delta x$ from the solution obtained using just one large time step of $2 \Delta t$ on a coarsened grid of $2 \Delta x$. Thus for a given point in the computational domain if the magnitude of Eq. (12) is larger than some specified amount (e.g. $\tau \geqslant$ threshold), then locally that region of the computational domain is refined (see [17].)

Such criteria in lower dimensional work have been demonstrated to work very well in demanding circumstances (e.g. $[18,22,25]$.) However, applying the truncation error estimator Eq. (12) as the refinement criteria, verbatim, can fail.

To understand what we mean by "fail," consider the $r a$ dial scaling of the waves as they propagate away from the black hole and the impact of this effect on the refinement criteria. The perturbed metric variables have strong radial falloff behavior (for sufficiently large radii $r, h \sim 1 / r$ ). In our single wave equation, this is very clear, although in a more general case, as one would encounter in the full set of Einstein equations, there will be a mixture of different falloff rates depending on the variables, the gauge, and so forth. But physically the wave part of the solution, given by $h$, will decay as it propagates away. Since refinement criteria like Eq. (12) are proportional to the local magnitude of the solution, the decaying nature on the function being refined undermines the effectiveness of the refinement criteria's ability to place refinement.

\section{Adaptive refinement studies for a pure $\ell=2$ mode}

We illustrate many of these issues by considering the effect of different refinement criteria on a 3D simulation for a pure $\ell=2$ wave, as discussed in the section above. We consider a general packet containing many $\ell$-modes, taking advantage of our generalized wave equation, in the next section. We note that these simulations are much more demanding than those discussed in the section above, where waveforms were measured near the peak of the potential near the horizon. In this section we study the propagation of waves far from the black hole, and our ability to dynamically track and resolve them.

Consider the refinement criteria Eq. (12) with the demand that $\tau \leqslant 0.002$ throughout a computation domain which extends to $300 M$ and a base resolution of $2 M$. In this simulation of the pure $\ell=2$ mode, we allow one level of refinement. In Fig. 7 we show the growth number of grid points for several adaptive mesh refinement simulations. As the waves propagate out from the black hole, they of course sweep out an ever larger volume. Plotting the percentage growth of dynamic grid points, relative to the number of

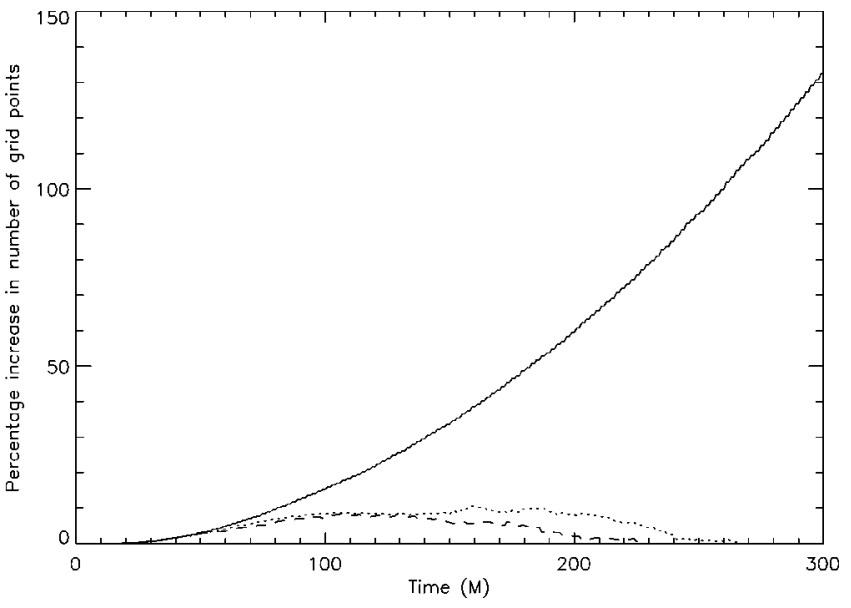

FIG. 7. Using the truncation error estimator Eq. (12) without radial scaling reveals that the refined region "evaporates" as it propagates outward (dotted line). This behavior is also true with the norm refinement criteria Eq. (13) (dashed line). However, appropriately scaling the norm refinement criteria produces a continuous growth of dynamic gridpoints (solid line).

initial points, as a function of time (see dotted line in Fig. 7) we see that the growth of the dynamic refinement occurred only over a finite period of time, eventually evaporated even as the waves expand to larger volumes. This is due to the decay of the wave amplitude below the level at which it can be captured by the specified truncation error limit. This is contrary to our AMR requirements for capturing and transporting the physical waveform to some arbitrarily distant detector. The solid and dashed lines correspond to other refinement criteria discussed below.

Indeed the net effect of such evaporation on a waveform is to dramatically perturb it, since waves once initially captured within some region of mesh refinement will not remain contained and instead "leak" back across the dynamic refinement boundaries onto coarser resolutions. To demonstrate this behavior, in Fig. 8 we show the actual waveform obtained for the same simulation, again demanding that $\tau$ $\leqslant 0.002$ throughout the computation domain. Initially this results in the first two out-going modes being contained within the dynamic refinement, although with only one level of refinement, the wave is still not resolved enough. However, by the time these modes have passed a detector placed at $125 \mathrm{M}$, only the first mode remains captured within the refinement- the second mode now trails in its wake. Moreover, because the evaporation of the grid is non-smooth (see Fig. 7 dotted line), this has an effect of perturbing the waveform which becomes highly inaccurate. Here the solid line is the correct waveform obtained from 1D simulations in the tortoise coordinate. The dotted line is the truncation-error based AMR result, clearly revealing the deterioration of the second mode which now trails the dynamic mesh refinement. (For a discussion on the effects moving boundaries have on perturbing waves straddling mesh refinement, see [25].) One cannot cure this effect by simply tightening the tolerance on the refinement criterion, since this can only improve things for awhile, but eventually as wave decays away, the same effects will be seen. 


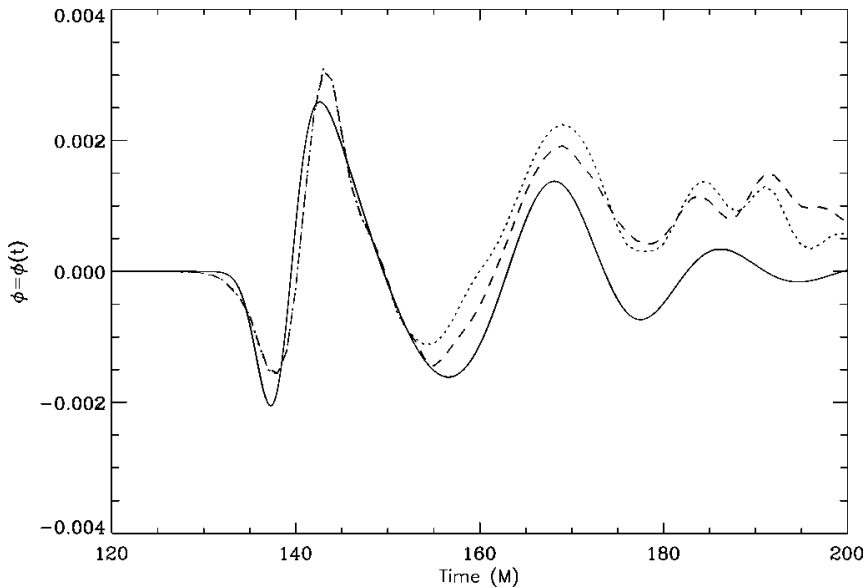

FIG. 8. Tracking of outgoing waves using one level of dynamic refinement. The signal as seen by an equatorial observer located at $125 \mathrm{M}$. The base grid resolution is $2 \mathrm{M}$. Using truncation error refinement criteria [Eq. (12)] with no radial scaling produces a poor quality signal (dotted line) compared to the 1D result (solid line). The trailing part of the signal falls outside the AMR captured region and deteriorates considerably. This is also the case for the norm refinement criteria (Eq. 13) with no radial scaling, shown as a dashed line.

Such behavior should not be thought of as indicative of truncation error estimators. This dynamic refinement behavior is generic to refinement criteria which do not take into consideration the underlying nature of the solution's falloff. For example, employing a refinement criterion based on a norm of the solution:

$$
N_{1}=c_{1}|\Phi|^{2}+c_{2}|\Phi, t|^{2},
$$

produces similar effects, as shown by the dashed lines in Fig. 7 and Fig. 8. In Fig. 7 we see the initial growth and final decay of dynamic grid points, and in Fig. 8 we see a similarly poor waveform. Interestingly, however, the evaporation of the dynamic refinement created in this case is more regular and hence shorter lived than that for refinement criteria based on estimating the truncation error. This difference can be traced to the fact that we found the truncation error estimator Eq. (12) produced a "noisier" estimate of where refinement was required and was therefore less able to create a regular pattern of refinement than the norm based criterion.

To overcome this grid evaporation requires an appropriate scaling the refinement criteria. For example, for the norm refinement criteria by scaling it radially with $1 / r^{2}$ results in both the first and second outgoing modes remaining captured within the mesh refinement-in principle forever. (The effects of dispersion will eventually negate this effect however.) This is shown by the dotted line in Fig. 9. Here, the trailing part of the signal that falls outside the dynamic region of refinement has deteriorated considerably. The quality of the signal can be increased further by introducing a second level of dynamic mesh refinement as shown by the dashed line in Fig. 9. We obtained similar results by suitably scaling the truncation error criterion, although as before, with a slightly noisier solution.

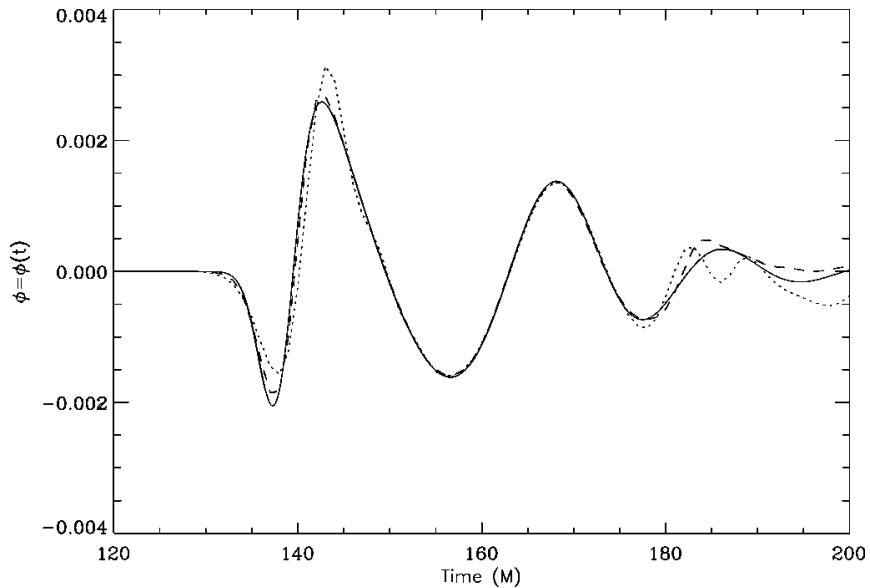

FIG. 9. Tracking of outgoing waves using one and two levels of dynamic refinement with a "scaled" refinement criterion. The signal shown is seen by an equatorial observer located at $125 \mathrm{M}$. The base grid resolution is $2 \mathrm{M}$. Using norm refinement criteria (Eq. 13) with $1 / r^{2}$ radial scaling captures and contains the first two outgoing modes (dotted line) compared to the $1 \mathrm{D}$ result (solid line). The trailing part of the signal falls outside the AMR captured region and has inevitably deteriorated. The quality of the signal improves even more when two levels of dynamic refinement are used (dashed line).

Finally, for simulations of a pure $\ell=2$ multipole wave hitting a black hole, we show the evolution of the grid refinement structure as the wave propagates out. Again we use a base grid extending out to $300 M$, with a resolution of $2 M$. Two levels of dynamic AMR track outgoing waves using the norm based refinement criteria [Eq. (13)] with the correct

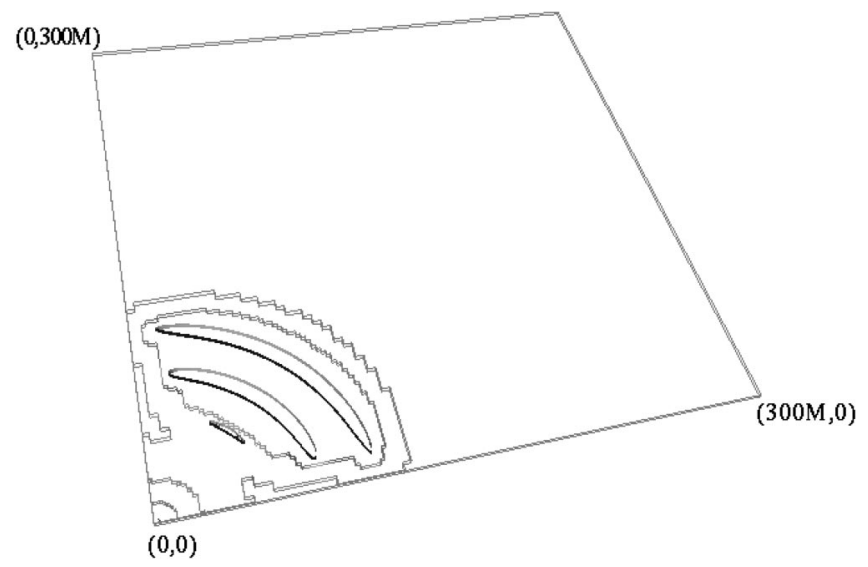

FIG. 10. Mesh structure and isosurfaces for an outgoing pulse, at time $100 \mathrm{M}$. The grid extends out to $300 \mathrm{M}$, with a base resolution of $2 \mathrm{M}$. The boundaries of the refinement layers are indicated by the zig-zag lines. Two such layers engulf the outgoing burst. Several layers cover the hole, of which only three are shown. At this stage the dynamically prescribed layers tracking the outgoing burst still overlap with the prescribed layers around the hole. Two wavelengths (depicted as isosurface shells) are captured by the $L_{2}$ refinement and will be propagated accurately outwards. A third oscillation is seen to be just outside $L_{2}$, a fact which will prove detrimental to its shape. 


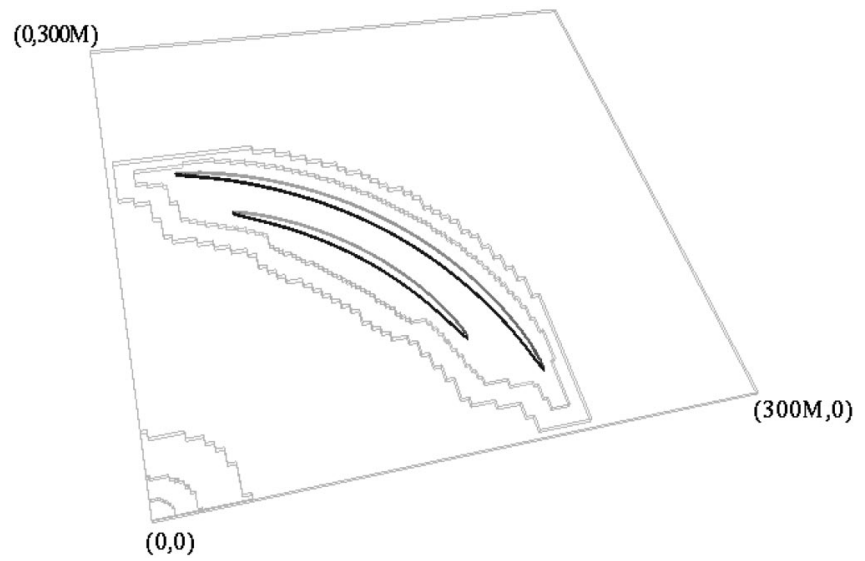

FIG. 11. Mesh structure and isosurfaces for outgoing pulse, at time 200M. The boundaries of the refinement layers covering the hole and the outgoing signal are clearly separated. The coarse region in-between cannot support the lower amplitude trailing signal, which will become heavily distorted.

radial scaling of $1 / r^{2}$. Seven layers of prescribed refinement resolve the region near the black hole horizon.

Figure 10 shows the mesh structure and isosurfaces for the outgoing pulse at time $t=100 \mathrm{M}$, and Fig. 11 show the same system at time $t=200 \mathrm{M}$. The boundaries of the refinement layers are indicated by the zig-zag lines. Two such layers engulf the outgoing burst and of the seven layers resolving the hole, only three are shown.

At the $t=100 M$ stage (Fig. 10) the dynamically prescribed layers tracking the outgoing burst still overlap with the prescribed layers around the hole. The two wavelengths (depicted as isosurface shells) are captured by the $\mathcal{R}_{2}$ refinement and will be propagated accurately outwards. The third oscillation resides just outside $\mathcal{R}_{2}$, a fact which will prove detrimental to its later accuracy. At $t=200 M$ (Fig. 11) the boundaries of the refinement layers covering the hole and the outgoing signal are clearly separated. The resulting coarse region in-between cannot support the lower amplitude trailing signal which consequently becomes heavily distorted.

In such simulations, with expanding wavefronts, the price one must now pay for keeping a solution correctly captured within 3D dynamic refinement is to inevitably have to increase the number of grid points within the computational domain. This is shown by the solid line in Fig. 7. This rapid growth of the consumed resources, as the wavefront expands onto the coarse grid, will be typical of the more general black hole coalescence problems. Thus, for the future effective use of AMR in black hole simulation, one must address directly the question of what amount of the signal needs to be captured with high resolution grids and set the refinement criteria accordingly (appropriately scaled by $r$ ) to distribute the refined grids around the strongest part of the signal, i.e., the initial burst, and the largest QNM oscillations immediately following.

\section{Simulations of general pulse hitting a black hole}

The previous discussion was based on a single initial data set, which contained only a pure $\ell=2$ angular structure. We

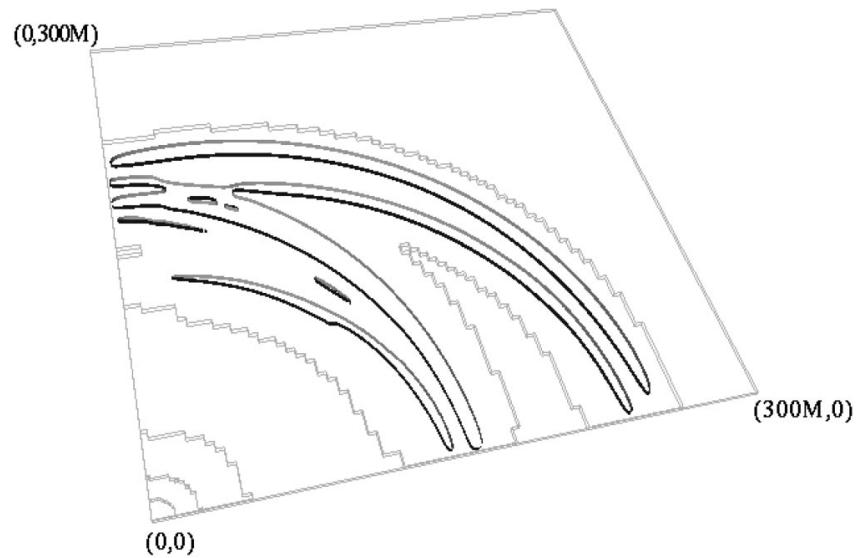

FIG. 12. Planar slice of mesh structure and isosurfaces of dumbbell shaped initial data, after $200 \mathrm{M}$ of evolution. A shell of overlapping high-frequency bursts will eventually be succeeded by the more regular pattern of QNM ringing.

turn now to a more general wave packet, probing the AMR performance on more complex wave patterns that could be encountered in the general black hole coalescence problem. To this end, we consider general initial data, for example data that have compact support in a three dimensional volume which does not surround the black hole, for example

$$
\begin{aligned}
& \Phi=e^{-\kappa\left[\left(\bar{x}-\bar{x}_{c}\right)^{2}+\left(\bar{y}-\bar{y}_{c}\right)^{2}+\left(\bar{z}-\bar{z}_{c}\right)^{2}\right]}, \\
& \dot{\Phi}=0 .
\end{aligned}
$$

It is obvious that such evolutions would be intrinsically three-dimensional. The emitted signal has a distinct "burst" phase, containing radiation of considerably high frequency. The presence of high harmonics, with the corresponding short wavelengths makes the accurate evolution of such data more demanding than simpler superpositions of low lying modes. Here we illustrate the ability of the AMR suite to capture complex solution patterns. The refinement criterion used was norm based [Eq. (13)] and one level of dynamic refinement.

Figure 12 shows a planar slice of mesh structure and isosurfaces of dumb-bell shaped initial data, after $200 \mathrm{M}$ of evolution. This "burst shell" of overlapping high-frequencies will eventually be succeeded by the more regular pattern of QNM ringing. Here, these high frequency features have no possibility of being accurately transported to a distant detector without dynamic AMR.

The corresponding three dimensional mesh structure at $\mathrm{t}=200 \mathrm{M}$ is shown in Fig. 13. Here octant symmetry, in connection with the initial data of compact support, produces "voids" in between the out-going wavefronts. This corresponds to the delayed arrival of the "mirror" data in the computational domain. In these regions dynamic refinement senses the absence of a strong signal and therefore leaves unrefined. 


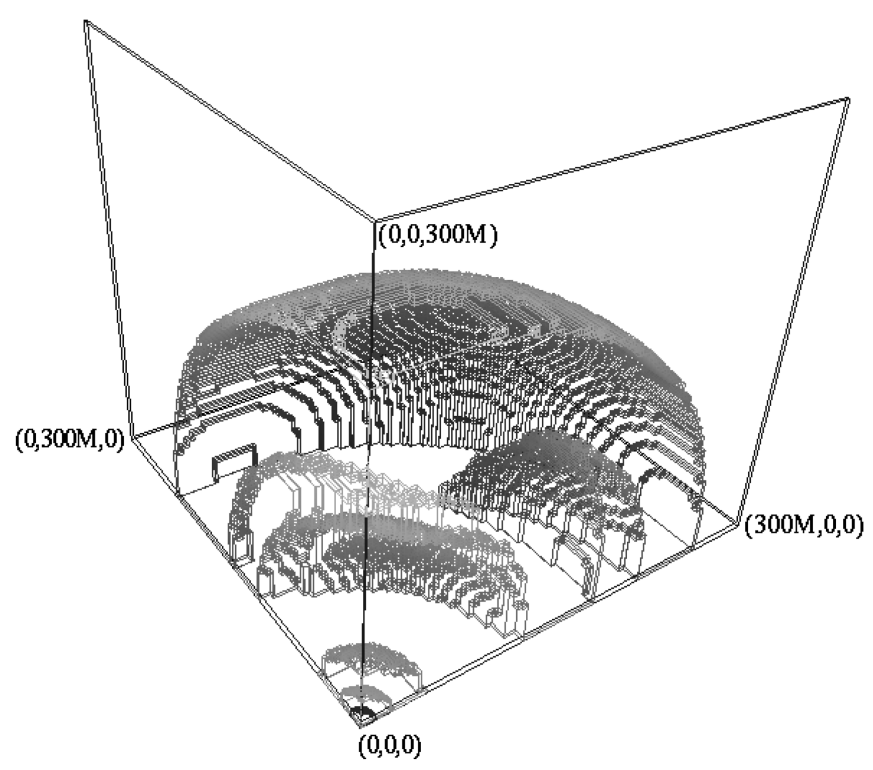

FIG. 13. Three dimensional mesh structure. The octant symmetry in connection with initial data of compact support produces "voids" in between the fronts, which correspond to the delayed arrival of the "mirror" data in the computational domain. The dynamic refinement senses the absence of strong signal and economizes the grid.

\section{CONCLUSIONS AND FUTURE DIRECTIONS}

This work presents an introduction to the potential and problems of full 3D adaptive mesh refinement in numerical relativity. For the purpose of our investigation in adaptive three dimensional computations, we introduced a general class of three-dimensional partial differential equations that capture important aspects of black hole interactions. For select parameters those equations correspond rather directly to physical models of scalar, electromagnetic and gravitational interactions of black holes, hence physical processes can be described (in the weak limit) in terms of those equations. Preliminary analysis of those equations outlines aspects of their behavior, in particular the dependence of the ring-down signal on the parameters, and the resolution requirements of the solution in the near horizon region in isotropic static slicings of the black hole.

We presented a series of fully resolved three-dimensional computations involving dynamics in black hole spacetimes. The linear nature of the problem does not reduce the large dynamic range of the black hole potential, which manifests itself through the strong radial dependence of the coefficients of the equation. Our adopted gauge accentuates the large resolution requirements near the horizon. Prescribed fixed refinement was successfully used to provide the required resolution in that region.

Dynamic refinement was used to propagate signals into the exterior domain. The necessary scaling of refinement criteria by appropriate power of $r$ was discussed, along with the impressive growth of grid points occurring when the outgoing burst is resolved dynamically as it propagates. The idea of norm-based refinement was introduced, which works well in conjunction with a selective tracking of the strongest part of the signal. Our considerations are based of course on our model equations, but may be useful for the Einstein problem. In particular we propose the (appropriately scaled) norm $N_{2}=c_{1}\left|\Psi_{4}\right|^{2}+c_{2}\left|\Psi_{4, t}\right|^{2}$, where $\Psi_{4}$ is a locally computed component of the Weyl tensor describing, in vacuum, outgoing radiation. This might prove to be an effective outer refinement criterion, and should be tested on model problems involving the full set of Einstein equations.

We are presently extending this work in several directions. Our study directly demonstrates that 3D investigations of black hole physics in the linearized limit can directly benefit from AMR methods. Extensions of the presently described models involving rotation and different stationary coordinate systems are underway [40]. Studies of non-linear systems are also underway (e.g., in connection with ADM evolution of single black hole spacetimes in threedimensions.) The considerable complexity of the adaptive mesh infrastructure suggests comparisons of the HHL data structure with the DAGH data structure, developed for the BBHGC alliance program, in particular with respect to performance in three-dimensional computations on parallel architectures.

\section{ACKNOWLEDGMENTS}

This work has been supported by the Albert Einstein Institute (AEI). Calculations were performed at AEI on an SGI/ Cray Origin 2000 supercomputer. We have benefited significantly from interactions with many colleagues at AEI and NCSA, especially Miguel Alcubierre, Gabrielle Allen, Bernd Brügmann, Randy Leveque, Joan Massó, Bernard Schutz, John Shalf, Bernd Schmidt, Wai-Mo Suen, and Paul Walker. The work presented was substantially complete, when we became aware of a recent paper [41] dealing with similar issues, but without adaptive mesh refinement. We acknowledge Andrew Abrahams and Luciano Rezzolla for illuminating discussions.

\section{APPENDIX: NUMERICAL ANGULAR MODE MIXING AND INSTABILITIES}

In this Appendix we elaborate on a feature of some of the Eqs. (4), which is important for stable long-term evolutions, a very desirable characteristic for a numerical problem.

The PDE (4) is equivalent to an $\ell$-sequence of separated one-dimensional PDE's. It is of some importance to note that from the point of view of approximate solutions to the initial value problem, the equivalence may break down. By this we mean that a discrete approximation to Eq. (4) will not (in general) be a separable difference equation, and hence numerical mixing of angular modes is possible, and is generally the case unless some techniques are devised to prevent it.

Some of the Eqs. (4) demonstrate in the late stages of numerical evolution a dramatic manifestation of such mode 
mixing. For values $K_{1}=-6, K_{2}=0$, the equation represents axial gravitational perturbations and hence should exclude non-radiative solutions with spherical or dipole symmetry $(\ell=0,1)$. It turns out that an approximate integration of the initial value problem is unstable with respect to the $\ell=0$ mode, i.e., even if the mode is absent in the initial data, it will appear in the solution and it will exhibit unbounded growth.

The origin of the unstable $\ell=0$ behavior can be easily seen by inspection of the separated Eq. (1) for $\ell=0$. The effective potential is negative in the entire domain, hence a simple examination of the dispersion relation $\left(\omega^{2}=\kappa^{2}-V\right)$ for spherical waves reveals the presence of local modes that are exponentially growing. Depending on the accuracy of the integration, the manifestation of the instability may be delayed, but in finite precision arithmetic, it is bound to occur due to round-off error. In our simulations it occurs typically after $100 \mathrm{M}$ of evolution for $128^{3}$ base-grids. It manifests itself much earlier if a spherical component is analytically introduced at the initial time.

For long time evolutions, with potentials admitting growing modes, excluding the unstable $\ell=0$ mode is possible with the appropriate use of boundary conditions. To this end, we restrict the integration domain to an octant, and select to impose at least one condition of anti-reflection across the planes defining the octant domain. Such con- ditions eliminate spherical modes even for the discretized equations. The spherical nature of the background conveniently allows one to rotate the coordinate system (this is of course the reason why multipoles of same $\ell$, but different $m$ values obey the same equations for non-rotating black holes).

As an example, the $\ell=2, m=0$ mode with angular dependence proportional to $3 \cos (\theta)^{2}-1$, will be decomposed, in a rotated frame $\hat{\theta}=\theta+\pi / 2$, to sum of quadrupole terms, of which the $\ell=2, m=2$ mode, with a further $\phi$ rotation by $\pi / 4$ gives $\sin (\hat{\theta})^{2} \cos (2 \hat{\phi}+\pi / 2)$. This angular dependence admits antireflection conditions across the $x=0$ and $y=0$ planes. Indeed, with the use of such conditions, the numerical integrations show no sign of unstable growth for at least $500 M$ of evolution time.

Axial perturbations will be present in full non-linear simulations, and their dynamics will be governed, at least in some weak regime, by a collection of coupled linear equations. It is not clear that such a linearized system of Einstein equations should have an unstable spherical mode of the same type exhibited by our model partial differential equations (PDE). This issue warrants some more investigation. If this were the case though, eliminating the instability would be very difficult for integrations in the full spatial domain and non-spherical holes.
[1] É. É. Flanagan and S. A. Hughes, Phys. Rev. D 57, 4535 (1998).

[2] É. É. Flanagan and S. A. Hughes, Phys. Rev. D 57, 4566 (1998).

[3] R. Gomez et al., Phys. Rev. Lett. 80, 3915 (1998).

[4] G. Allen, K. Camarda, and E. Seidel, "Evolution of Distorted Black Holes. A perturbative Approach,' gr-qc/9806014.

[5] G. Allen, K. Camarda, and E. Seidel, “3D Black Hole Spectroscopy: Determining Waveforms from 3D Excited Black Holes,"' gr-qc/9806036.

[6] K. Camarda, Ph.D. thesis, University of Illinois at UrbanaChampaign, Urbana, Illinois, 1998.

[7] K. Camarda and E. Seidel, Phys. Rev. D 57, 3204 (1998).

[8] P. Anninos, K. Camarda, J. Massó, E. Seidel, W.-M. Suen, and J. Towns, Phys. Rev. D 52, 2059 (1995).

[9] P. Anninos, J. Massó, E. Seidel, W.-M. Suen, and M. Tobias, Phys. Rev. D 56, 842 (1997).

[10] S. Chandrasekhar, The Mathematical Theory of Black Holes (Oxford University Press, Oxford, England, 1983).

[11] E. Seidel and W.-M. Suen, Phys. Rev. Lett. 69, 1845 (1992).

[12] G. B. Cook et al., Phys. Rev. Lett. 80, 2512 (1998).

[13] N. Bishop, R. Isaacson, R. Gomez, L. Lehner, B. Szilagyi, and J. Winicour, in On The Black Hole Trail, edited by B. Iyer and B. Bhawal (Kluwer Academic, Dordrecht, 1998), gr-qc/9801070.

[14] A. M. Abrahams et al., Phys. Rev. Lett. 80, 1812 (1998).

[15] P. Hübner, Phys. Rev. D 53, 701 (1996).

[16] R. Leveque and M. Berger, SIAM (Soc. Ind. Appl. Math.) J.
Numer. Anal. (to be published).

[17] M. Berger and J. Oliger, J. Comput. Phys. 53, 484 (1984).

[18] M. Choptuik, Phys. Rev. Lett. 70, 9 (1993).

[19] M. Choptuik, in Frontiers in Numerical Relativity, edited by C. Evans, L. Finn, and D. Hobill (Cambridge University Press, Cambridge, England, 1989).

[20] A. Abrahams and C. Evans, Phys. Rev. Lett. 70, 2980 (1993).

[21] R. Hamade and J. M. Stewart, Class. Quantum Grav. 13, 497 (1996).

[22] J. Massó, E. Seidel, and P. Walker, in The Seventh Marcel Grossmann Meeting: On Recent Developments in Theoretical and Experimental General Relativity, Gravitation, and Relativistic Field Theories, edited by R. T. Jantzen, G. M. Keiser, and R. Ruffini (World Scientific, Singapore, 1996), p. 634.

[23] M. Parashar and J. C. Browne, Proceedings of the International Conference for High Performance Computing 22 (1995).

[24] L. Wild and B. Schutz (in preparation).

[25] L. A. Wild, Ph.D. thesis, University of Wales, 1996.

[26] R. H. Price and J. Pullin, Phys. Rev. Lett. 72, 3297 (1994).

[27] P. Anninos, R. H. Price, J. Pullin, E. Seidel, and W.-M. Suen, Phys. Rev. D 52, 4462 (1995).

[28] A. Abrahams and R. Price, Phys. Rev. D 53, 1972 (1996).

[29] J. Baker, A. Abrahams, P. Anninos, S. Brandt, R. Price, J. Pullin, and E. Seidel, Phys. Rev. D 55, 829 (1997).

[30] R. J. Gleiser, C. O. Nicasio, R. H. Price, and J. Pullin, Phys. Rev. Lett. 77, 4483 (1996). 
[31] Here we show that computational relativists can always manage to use a supercomputer for what mathematical relativists can do with "pencil and paper."

[32] T. Regge and J. Wheeler, Phys. Rev. 108, 1063 (1957).

[33] R. Price, Phys. Rev. D 5, 2419 (1972).

[34] S. Brandt and E. Seidel, Phys. Rev. D 52, 870 (1995).

[35] W. Krivan, P. Laguna, and P. Papadopoulos, Phys. Rev. D 54, 4728 (1996).

[36] W. Krivan, P. Laguna, P. Papadopoulos, and N. Andersson, Phys. Rev. D 56, 3395 (1997).

[37] R. Gomez, J. Winicour, and B. Schmidt, Phys. Rev. D 49, 2828 (1994).
[38] B. Schmidt, in Proceedings of the 4th Hungarian Relativity Workshop, edited by S. Perjes (unpublished).

[39] M. Berger and I. Rigoutsos, IEEE Trans. Syst. Man Cybern. 21 (1991).

[40] P. Papadopoulos and J. A. Font, Phys. Rev. D 58, 024005 (1998).

[41] L. Rezzolla, A. M. Abrahams, T. W. Baumgarte, G. B. Cook, M. A. Scheel, S. L. Shapiro, and S. A. Teukolsky, Phys. Rev. D 57, 1084 (1998).

[42] B. Brügmann, gr-qc/9708035 (1997).

[43] L. Wild and P. Walker (in preparation). 